\title{
Rapid fabrication of pressure-driven open-channel microfluidic devices in omniphobic RF paper
}

\section{Citation}

Glavan, Ana C., Ramses V. Martinez, E. Jane Maxwell, Anand Bala Subramaniam, Rui M. D. Nunes, Siowling Soh, and George M. Whitesides. 2013. "Rapid Fabrication of Pressure-Driven Open-Channel Microfluidic Devices in Omniphobic RF Paper." Lab Chip 13, no. 15: 2922-2930.

\section{Published Version}

doi:10.1039/c3lc50371b

\section{Permanent link}

http://nrs.harvard.edu/urn-3:HUL.InstRepos:12362621

\section{Terms of Use}

This article was downloaded from Harvard University's DASH repository, and is made available under the terms and conditions applicable to Open Access Policy Articles, as set forth at http:// nrs.harvard.edu/urn-3:HUL.InstRepos:dash.current.terms-of-use\#OAP

\section{Share Your Story}

The Harvard community has made this article openly available.

Please share how this access benefits you. Submit a story.

Accessibility 


\title{
Rapid Fabrication of Pressure-driven Open-Channel Microfluidic Devices in Omniphobic $\mathbf{R}^{\mathrm{F}}$ Paper
}

\author{
Ana C. Glavan, ${ }^{\text {a: }}$ Ramses V. Martinez, ${ }^{\text {a }}$ E. Jane Maxwell, ${ }^{\text {a }}$ Anand Bala Subramaniam, \\ Rui M.D. Nunes, ${ }^{a}$ Siowling Soh, ${ }^{a}$ and George M. Whitesides ${ }^{\mathrm{a}, \mathrm{b}^{\mathrm{t}}}$ \\ ${ }_{5}$ Received (in $\left.X X X, X X X\right)$ Xth $X X X X X X X X X 20 X X$, Accepted Xth XXXXXXXXX 20XX \\ DOI: $10.1039 / b 000000 x$
}

This paper describes the fabrication of pressure-driven, open-channel microfluidic systems with lateral dimensions of 45-300 microns carved in omniphobic paper using a craft-cutting tool. Vapor phase silanization with a fluorinated alkyltrichlorosilane renders paper omniphobic, but preserves its high gas 10 permeability and mechanical properties. When sealed with tape, the carved channels form conduits capable of guiding liquid transport in the low-Reynolds number regime (i.e. laminar flow). These devices are compatible with complex fluids such as droplets of water in oil. The combination of omniphobic paper and a craft cutter enables the development of new types of valves and switches, such as "fold" valves and "porous switches," which provide new methods to control fluid flow.

\section{${ }_{15}$ Introduction}

Micro paper-based analytical devices ( $\mu$ PADs), ${ }^{1-7}$ in which aqueous solutions wick through a porous matrix of hydrophilic cellulose fibers delimited by hydrophobic barriers, provide an alternative to elastomer (PDMS) and rigid polymer-based, open20 channel microfluidic systems. ${ }^{8}$ Their relatively low cost and simplicity have made $\mu$ PADs attractive for prototyping concepts in microanalysis, and perhaps in applications in areas such as medical diagnostics for resource-limited environments, ${ }^{9-15}$ environmental monitoring, ${ }^{16-19}$ food safety testing, ${ }^{20}$ and forensic

25 analysis. ${ }^{18,19}$ Despite their advantages, current paper microfluidic technologies share some common disadvantages: limited minimum feature sizes (channel widths are generally greater than $200 \mu \mathrm{m})$ and inefficient delivery of sample within the device. Due to sample retention in the porous cellulose matrix, the 30 volume that reaches the detection zones is usually less than $50 \%$ of the total volume within the device. ${ }^{7}$ Moreover, applications that require low Reynolds number, pressure-driven flows in open channels-for example, multiphase flows involving drops or bubbles, or flows of complex fluids such as whole blood or ${ }_{35}$ colloidal suspensions that contain particulates - are generally incompatible with wicking flow.

Here we describe the use of a craft-cutting tool to fabricate pressure-driven microfluidic devices with feature sizes as small

${ }_{40}{ }^{a}$ Department of Chemistry and Chemical Biology, Harvard University, 12 Oxford Street, Cambridge, MA 02138, USA.

${ }^{b}$ Wyss Institute for Biologically Inspired Engineering, Harvard University, 60 Oxford Street, Cambridge, MA 02138, USA.

$\$$ Authors have contributed equally to this work.

$45\left(^{*}\right)$ Author to whom correspondence should be addressed: gwhitesides@gmwgroup.harvard.edu

$\dagger$ Electronic Supplementary Information (ESI) available. See DOI: $10.1039 / \mathrm{b} 000000 \mathrm{x} /$ as $45 \mu \mathrm{m}$, using, as matrix for fabrication, omniphobic $\mathrm{R}^{\mathrm{F}}$ paper 50 prepared by silanization of cellulose paper with a fluoroalkyl silanizing reagent. ${ }^{21}$ The entire process of design and fabrication can be completed in less than ten minutes. These devices display low-Reynolds number fluid dynamics (i.e. laminar flow), make possible new types of simple valves and switches to control fluid 55 flow, and exhibit high gas permeability.

Analytical systems using microfluidics have been actively explored since the late 1980s. The introduction of fast prototyping techniques based on soft lithography ${ }^{8}$ led to increased research and development of polymer-based microfluidic 60 systems, but the fabrication of these devices still requires specialized equipment, moderately expensive polymeric materials, and significant expertise.

A range of alternative techniques for the fabrication of microfluidic devices in polymeric sheets have been demonstrated; ${ }_{65}$ these techniques include transfer masking of toner, ${ }^{22}$ patterning of double-sided tape or film with a laser $\operatorname{cutter}^{23,24}$ or cutting plotter, ${ }^{25,} 26$ UV-initiated surface photografting to pattern superhydrophilic channels on the surface of a thin superhydrophobic porous polymer film, ${ }^{27}$ or embossing channels

70 into polymeric sheets using a rigid master at elevated temperature and pressure. $^{28}$ Linder, Sia et al. used injection molding to fabricate microfluidic cassettes from polystyrene and cyclic olefin copolymers. $^{29-31}$

$\mu$ PADs generally use hydrophobic barriers patterned in paper 75 to define paths along which liquids can wick. Methods for patterning paper include the use of a photoresist (SU-8) and photolithography, ${ }^{1,} 32-34$ wax printing, ${ }^{2}, 35$ or printing of polydimethylsiloxane (PDMS) dissolved in hexanes. ${ }^{36}$ Other fabrication techniques begin with paper already coated with a 80 hydrophobic layer on which specific areas are then plasma etched, ${ }^{37}$ dissolved, ${ }^{6}$ or laser-treated ${ }^{38}$ in order to define 
hydrophilic regions. When no hydrophobic barriers are present, the shape of the paper itself defines the fluid path. Fenton et al. ${ }^{39}$ pioneered the use of a computer-controlled XY-knife plotter to shape thin sheets of paper and other porous media to define fluid 5 paths. The use of shaped paper has subsequently been developed by others. ${ }^{40-46}$

This paper describes a bench-top fabrication process that integrates the common elements of pressure- driven microfluidics (e.g. laminar flow, mixing, on/off valves, gradient and droplet 10 generators) in a system that uses omniphobic paper as a substrate. These easy-to-prototype, inexpensive, pressure-driven devices expand the repertoire of microfluidic manipulations and analyses that can be conducted using paper, and offer a useful new method for the fabrication of microfluidic devices.

\section{${ }_{15}$ Materials and Methods}

\section{Choice of materials}

We used an electronic craft cutter (Silhouette America, Inc., Silhouette Cameo ${ }^{\mathrm{TM}}$ ), with either a thin cutting blade or an engraving tip, to carve micro-channels into the surface of a sheet 20 of cardstock paper. Craft cutters are inexpensive ( $\$ 150-300)$, can reproduce channels designed using simple software, are safe, and are extremely easy to use. We chose cardstock paper as the material for cutting because it is lightweight and flexible, sufficiently smooth to create a tight seal with tape, and 25 inexpensive ( $\sim 0.06$ for an $81 / 2 " \mathrm{~N} 11$ " sheet); it is also thick enough $(300 \mu \mathrm{m})$ to retain mechanical stability while accommodating the depths of the carved channels.

Silanization of paper with an alkyl or fluoroalkyl trichlorosilane makes it hydrophobic; the reaction occurs readily 30 with the silanizing agent in the vapor phase, and requires no equipment apart from a low-pressure chamber and a source of heat. ${ }^{21}$ The cost of the materials used for functionalization with a long fluoroalkyl trichlorosilane is $\sim \$ 0.80$ per $\mathrm{m}^{2}$ of paper (for reagents purchased in small quantities), slightly less than 35 rendering paper hydrophobic by wax printing $\left(\$ 1\right.$ per $\left.\mathrm{m}^{2}\right){ }^{2}$ The silanization treatment does not degrade the physical properties of the paper, and does not require pre- or post- treatment steps (e.g. washing to remove reagents or side products, drying, etc.). We chose $3,3,4,4,5,5,6,6,7,7,8,8,9,9,10,10,10$-heptadecafluorodecyl40 trichlorosilane, $\mathrm{CF}_{3}\left(\mathrm{CF}_{2}\right)_{7} \mathrm{CH}_{2} \mathrm{CH}_{2} \mathrm{SiCl}_{3} \quad\left(\mathrm{C}_{10}{ }^{\mathrm{F}}\right)$, and decyltrichlorosilane, $\mathrm{CH}_{3}\left(\mathrm{CH}_{2}\right)_{9} \mathrm{SiCl}_{3} \quad\left(\mathrm{C}_{10} \mathrm{H}\right)$ because they are commercially available, volatile, and reactive toward the hydroxyl groups of cellulose. This silanization reaction generates highly hydrophobic surfaces on the cardstock paper (static 45 contact angles of water $\theta_{\mathrm{s}}{ }^{\mathrm{C} 10 \mathrm{~F}}=137^{\circ} \pm 4^{\circ}, \mathrm{n}=20$ and $\theta_{\mathrm{s}}{ }^{\mathrm{C} 10 \mathrm{H}}=131^{\circ}$ $\pm 5^{\circ}, \mathrm{n}=20$ ). Paper functionalized with $\mathrm{C}_{10} \mathrm{~F}$ is also oleophobic (contact angle with hexadecane $\theta_{\mathrm{s}}{ }^{\mathrm{C} 10 \mathrm{~F}}=93 \pm 3^{\circ}, \mathrm{n}=10$ ). In contrast, paper functionalized with $\mathrm{C}_{10}{ }^{\mathrm{H}}$ is wet by hexadecane. We silanized the paper after carving the microfluidic channels,

50 rather than before, in order to avoid damaging the organosilane layer or exposing cellulose fibers that had not come in contact with vapors of organosilane.

Tape (PET/EVA/LDPE, Fellowes adhesive sheet, http://www.amazon.com/Fellowes-Self-Adhesive-Sheets-Letter5 ${ }_{55} 221502 / \mathrm{dp} / \mathrm{B} 0010 \mathrm{~K} 824 \mathrm{~A}$ ) provides a low-cost, optically transparent seal for the microfluidic channels. We used a syringe pump, connected to the device through reusable silicone tubing (1.57 mm OD), flangeless ferrules, and rings of double-sided adhesive layer (3M Command Medium Picture Hanging Strips, ${ }_{60} \mathrm{http} / /$ www.command.com/wps/portal/3M/en_US/NACommand/ Command/Products/Catalog/?N=3294736519\&rt=rud), to drive flow through the omniphobic channels. For applications requiring hexadecane, which can dissolve the double-sided adhesive layer, the ferrules were connected to the device using Instant Krazy ${ }_{65}$ Glue (Krazy Glue, Columbus OH).

Fabrication of open-channel paper microfluidic devices using an electronic craft cutter

Fig.1 schematically outlines the procedure used to fabricate pressure-driven paper microfluidic devices. We created a 70 blueprint for the microchannels using computer-aided design software (Adobe Illustrator ${ }^{\circledR}$ CS5, Adobe Systems Inc.) and carved the microchannels into the surface of the paper substrate using an electronic craft cutting/engraving tool. We rendered the resulting paper hydrophobic by vapor phase functionalization 75 with $\mathrm{C}_{10}{ }^{\mathrm{F}} \cdot{ }^{21}$ Following functionalization, we sealed the top of the device using a layer of tape with pre-cut holes aligned with the inlets and the outlets of the microchannels. We connected these channels to silicone tubing using flangeless ferrules affixed to the device with rings of double-sided adhesive tape. A syringe pump 80 drove fluid from the inlets to the outlets of the open microchannels at flow rates of $5-100 \mu \mathrm{L} / \mathrm{min}$. For applications requiring a fixed inlet pressure, rather than a fixed volumetric flow rate, we used gravity-driven flow and adjusted the hydrostatic pressure by controlling the height of the inlet ${ }_{85}$ reservoir with respect to the waste reservoir. These microfluidic devices can withstand hydrostatic pressures up to 0.27 bar $(27 \mathrm{kPa})$ without delaminating.

The complete sequence of fabrication steps from design concept to a working device can be completed within ten 90 minutes: (i) carving of 40 simple individual devices in a sheet of cardstock paper takes less than 30 seconds (ii) vapor-phase silanization of the entire engraved sheet requires $\sim 5$ minutes, and (iii) the assembly of one device (i.e. affixing adhesive layers and tubing) takes $\sim 2$ minutes.

95 Optimizing the quality of the seal between the inlets of the device and the fluid supply is important for minimizing the probability of failure of the devices (here we define success as the continuous flow of liquid from inlet to outlet under a constant pressure of $\sim 0.2$ bar ( $20 \mathrm{kPa}$ ) for an observation time of at least 100 one hour without leakage or delamination occurring at fluidic connections or along the fluidic path). To seal the ferrule to the paper device, we used a strong double-sided adhesive tape (3M Command Medium Picture Hanging Strips); no leakage at the ferrule occurred during our observation time (65/65 cases). ${ }_{105}$ On a few occasions (2/65), fragments of cellulose fiber or dust trapped in the channel during the fabrication process impeded the flow of liquids through the microfluidic device. Movie $\mathrm{S} 1 \uparrow$ shows the continuous flow of an aqueous solution through an open microfluidic channel engraved in omniphobic $\mathrm{R}^{\mathrm{F}}$ paper, as 110 observed over a period of 3 hours.

\section{Results and Discussion}


Laminar flow and passive mixing in open paper microchannels

To demonstrate the versatility of this fabrication process, we created channels of different widths by choosing appropriate 5 blades to use with the craft-cutting machine: a thin blade generated channels with widths of $45 \pm 5 \mu \mathrm{m}(\mathrm{n}=5$, based on SEM images), whereas an engraving tip generated channels with widths of 100 to $300 \mu \mathrm{m}$. In both cases, selecting appropriate settings of the craft-cutter can produce microchannels with depths 10 between 50 and $300 \mu \mathrm{m}$. The dimensions of the channels can be controlled by the combination of tip width and craft-cutter settings.

Fig. 2C, E shows a pressure-driven paper microfluidic device exhibiting laminar flow: two miscible aqueous phases, each 15 labeled with a different water-soluble dye $(0.05 \%$ solutions of Methylene Blue or Congo Red in water), were pumped through a $\mathrm{T}$-junction at a flow rate of $10 \mu \mathrm{L} / \mathrm{min}$ (Reynolds number $R e=2$ ). An optical microscope at 50x magnification imaged the two parallel streams within the $45-\mu \mathrm{m}$-wide channel (see inset in Fig. $202 \mathrm{E}$ ). These observations confirm that these devices can reproduce the classical diffusion-limited co-flows reported in open-channel microfluidic devices fabricated in materials such as PDMS. ${ }^{47}$

To produce a device capable of mixing two fluid streams at low Reynolds number, we carved a serpentine channel-a ${ }_{25}$ channel geometry that induces mixing between two co-flowing liquids ${ }^{48}$-into the paper (Fig. 2D, F). Two separate fluid streams $(0.05 \%$ solutions of Tartrazine or Methylene Blue in water) entered through a Y-junction at a flow rate of $10 \mu \mathrm{L} / \mathrm{min}$. Repeating C-shaped units turned the fluid through $180^{\circ}$ to induce 30 chaotic advection and passively enhance the mixing of the streams. Thus close to the inlet we observed two clearly delineated blue and yellow co-flowing liquid streams that then became a single stream of green liquid $18 \mathrm{~mm}$ downstream from the inlet (Fig. 2F). The mechanism for inducing chaotic 35 advection is the consecutive generation of Dean vortices in the curved microchannel. ${ }^{48}$

\section{Regulation of fluid flow by folding the microchannel}

Valving systems developed for elastomer-based microfluidic systems are compatible with pressure-driven paper microfluidic

${ }_{40}$ devices. For example, twist valves ${ }^{49}$ can turn the fluid flow on/off in paper microchannels (see Fig. S5, ESI †े).

We took advantage of the ease with which paper can be folded to devise an alternative procedure for valving that does not require the use of external parts. These "fold" valves reduce the

${ }_{45}$ flow rate through the microfluidic channel when the paper device is folded along an axis perpendicular to the fluid flow. They are probably not applicable to complex problems in sequential valving (as, for example, the sort of systems developed by Quake and others ${ }^{50,51}$ ), but provide a simple solution for controlling 50 flows in elementary systems.

We characterized the "fold" valve by measuring the amount of liquid expelled from the outlet as a function of the dihedral angle of the folded paper making up this valve. We adjusted the height of the liquid reservoir to obtain a steady flow of $\sim 20 \mu \mathrm{L}$ per 55 minute for the unfolded device. We then adjusted the dihedral angle while simultaneously wiping away any excess liquid at the outlet. After $60 \mathrm{~s}$, we used a calibrated micropipette to measure the volume of liquid expelled as a function of the dihedral angle. Fig. 3 illustrates the relationship between the dihedral angle at the ${ }_{60}$ crease and the amount of liquid expelled at the outlet. Increasing the dihedral angle resulted in a continuous decrease in the amount of liquid expelled at the outlet; no liquid was expelled at the outlet when the folding angle reached or exceeded $90^{\circ}$ (Fig. 3A). Unfolding the crease restored the flow along the fluid path (Fig. ${ }_{65} 3 \mathrm{~B}$ ). Each valve could be closed/opened at least ten times. Although we have not characterized in detail the mechanism by which this valve operates, we speculate that the tape constricts the channel as the paper is creased (Fig. S7, ESI $\uparrow$ ), and decreases the rate of flow through the channel. We expect the "fold" valve 70 to be able to withstand pressures up to $\sim 27 \mathrm{kPa}$, since at that pressure, the devices fail through delamination or permeation of liquid into the porous hydrophobic matrix.

\section{Regulation of fluid crossover between channels by pressure difference}

75 Omniphobic paper can also function as a "porous valve". Because the silanized paper fibers have a low surface free energy, liquid water does not spontaneously enter the pores of the hydrophobic paper (i.e. water does not wick into the paper due to capillarity). An applied pressure difference, however, should be 80 able to force water through the hydrophobic paper pores.

The pressure difference, $\Delta P_{\gamma}$, required to overcome the surface free energy of the hydrophobic surface of the pores of radius, $R$, is given by the Young-Laplace Equation (Eq. 1), where $\theta_{S}{ }^{22 O}$ is the static contact angle of water with the surface:

$$
\Delta P_{\gamma}=-\frac{2 \gamma \cos \theta_{S}^{H_{2} O}}{R}
$$

For our system, in which $\theta_{S}^{H 2 O} \sim 137^{\circ}, \gamma=72 \mathrm{mN} / \mathrm{m}$, and $R \sim$ $2.6 \mu \mathrm{m}$ (estimated from SEM images), Eq. 1 predicts that a difference in pressure $\Delta P_{\gamma}$ of $26 \mathrm{kPa}$ is required for water to break through the hydrophobic pores. This value is-perhaps ${ }_{95}$ coincidentally - that at which the escape of water from the channel into the hydrophobic pores of the surrounding $\mathrm{R}^{\mathrm{F}}$ paper matrix is observed.

The pressure difference $(\Delta P)$ required to move water a distance $\mathrm{L}$ through a porous medium, such as paper, is given by 100 Darcy's equation (Eq.1), where $Q$ is the volumetric rate of flow through the porous medium $\left(\mathrm{m}^{3} / \mathrm{s}\right), k$ is the permeability of the medium $\left(\mathrm{m}^{2}\right), A$ is the cross sectional area to flow $\left(\mathrm{m}^{2}\right)$, and $\mu$ is the viscosity of the liquid $(\mathrm{Pa} \cdot \mathrm{s})$.

$$
\Delta P_{\mu}=\frac{\mu L Q}{k A}^{105}
$$

The total pressure needed to initiate flow and drive water across the porous medium of length $L$ can be approximated as the sum 110 of the pressures calculated in equations 1 and 2:

$$
\Delta P_{\gamma}=-\frac{2 \gamma \cos \theta_{S}^{H_{2} O}}{R}+\frac{\mu L Q}{k A}
$$

The porous valve is "closed" below this threshold pressure. 115 When the pressure exceeds the threshold value, the valve "opens" and water is forced through the pores of the paper.

Fig. 4A shows a diagram of a microfluidic device that uses a pressure-dependent porous valve to direct water into one of two 
different paths depending on the location and magnitude of the applied pressure. Two nonintersecting channels were designed such that the shortest distance between the channels was $0.8 \mathrm{~mm}$. We sealed both sides of the device with gas-impermeable tape to 5 allow the application of a vacuum through the paper channel (the paper devices discussed in the previous sections were sealed with tape on only one side) and placed a drop of an aqueous solution of dye at the inlet of the first channel to serve as a reservoir. Application of a vacuum $(\sim 5 \mathrm{kPa})$ at outlet 1 caused the water to 10 flow from the inlet to this outlet. Application of a vacuum at outlet 2 changed the flow path: when the vacuum exceeded a threshold pressure $(\sim 300 \pm 30$ Torr, or $\sim 40 \mathrm{kPa})$, the water passed through the hydrophobic paper in the region separating the two channels (Fig. 4), following the path from the inlet to outlet 2.

15 Below the threshold pressure, no water passed through the hydrophobic paper; the paper served as a porous valve with an opening pressure of $40 \mathrm{kPa}$. This pressure difference represents the threshold pressure required for water to overcome both the surface free energy $(\Delta P=26 \mathrm{kPa})$ and resistance to flow through 20 the pores of cardstock paper functionalized with $\mathrm{C}_{10}{ }^{\mathrm{F}}\left(\Delta P_{\mu}=40-\right.$ $26=14 \mathrm{kPa}$ ). Since the liquid passed though a bulk porous medium with no barriers to guide the flow, some liquid permeated the pores laterally and caused an apparent "bulge" at the crossing over between the channels. This "bulge" was

${ }_{25}$ consistently observed across a range of distances $L$ and threshold pressures $P$.

We measured the linear velocity of water infiltrating the channel corresponding to outlet 2 at a pressure of $40 \mathrm{kPa}$, and estimated a volumetric flow rate $Q=10^{-11} \mathrm{~m}^{3} / \mathrm{s}$ based on the 30 cross-sectional area $A=50 \times 150 \mu \mathrm{m}^{2}$ of the paper channel. Using Equation 2, we obtained a value for the permeability of the paper, $k$ of $\sim 10^{-14} \mathrm{~m}^{2}$. This value is in agreement with other results from the literature on the permeability of paper. ${ }^{52}$ Thus, by examining Equation 2, porous valves in paper devices can be 35 designed to have a range of opening pressures. For example, valves with higher threshold pressures can be designed using paper with lower porosity and, thus, lower permeability, $k$.

\section{Gas transfer between adjacent independent microchannels}

Paper - a fibrous matrix containing a network of interconnected 40 pores-generally exhibits much higher permeability to gas than the solid materials used to fabricate microfluidic devices. It is, for example, more than 100 times more permeable to oxygen than PDMS, which is itself unusually permeable (see Table S1, ESI $\dagger$, for other materials). We used the high permeability of paper to 45 enable rapid gas transport $(<1 \mathrm{~s})$ between two parallel microfluidic channels, the first of which contained a solution of dissolved $\mathrm{HCl}$ or $\mathrm{NH}_{3}$, while the second contained an indicator for the volatile compound present in the first channel.

We passed saturated aqueous solutions of dissolved $\mathrm{HCl}$ or ${ }_{50} \mathrm{NH}_{3}$ (37\% and 28\%, respectively) through one channel, which was parallel to a second channel containing a solution of universal $\mathrm{pH}$ indicator (Fig. 5A). The devices were sealed with gas-impermeable tape on both sides. The color of the $\mathrm{pH}$ indicator in the channel parallel to the $\mathrm{HCl}$ stream changed from 55 blue to yellow (from $\mathrm{pH} 9$ to $\mathrm{pH}$, Fig. 5C), while the color of the $\mathrm{pH}$ indicator in the channel parallel to the $\mathrm{NH}_{3}$ stream changed from green to blue (from $\mathrm{pH} 7$ to $\mathrm{pH} 10$, Fig. 5E). These color changes occurred within less than a second of the liquids filling the channels. We observed no change in the color of the ${ }_{60} \mathrm{pH}$ indicator when we repeated this procedure with aqueous solutions of a non-volatile acid $\left(37 \% \mathrm{H}_{2} \mathrm{SO}_{4}\right)$ or base $(8 \% \mathrm{NaOH}$, which has the same $\mathrm{pH}=13.6$ as the $28 \%$ solution of ammonia) in the neighboring channel (Fig. S5, ESI $\dagger$ ).

We interpret these results to indicate that the diffusion of ${ }_{65}$ vapors of the volatile acid or base $\left(\mathrm{HCl}\right.$ or $\left.\mathrm{NH}_{3}\right)$ through the walls of the microfluidic device causes a change in the $\mathrm{pH}$ of the solution in the neighboring channel. Our interpretation is supported by separate tests in which the omniphobic paper generated by functionalization with a fluoroalkane $\left(\mathrm{C}_{10}{ }^{\mathrm{F}}\right)$ resisted 70 wetting by concentrated solutions of $\mathrm{HCl}, \mathrm{H}_{2} \mathrm{SO}_{4}, \mathrm{NH}_{3}$, or $\mathrm{NaOH}$ applied on its surface for more than two hours (the duration of our observation; data not shown). The timescale of the observed color change $(<1 \mathrm{~s})$ is also consistent with that expected for the gaseous diffusion of molecules between the channels, estimated 75 by Eq. 4, where $\mathrm{L}$ is the distance (in one-dimension), $\tau$ is time (s), and $D$ is the diffusion constant of molecules of gas.

$$
\tau \sim \frac{L^{2}}{2 D}
$$

80 The diffusion constant, $\mathrm{D}$, of small gaseous molecules such as $\mathrm{NH}_{3}$ is typically on the order of $10^{-6} \mathrm{~m}^{2} / \mathrm{s}$. Thus, it should take $\sim 0.5 \mathrm{~s}$ for the $\mathrm{NH}_{3}$ vapor to diffuse between the channels.

The high gas permeability of paper also allows the removal of gas contaminants and unwanted air bubbles, which diffuse 85 through the walls of the device. We demonstrate the removal of air bubbles as large as $80 \mu \mathrm{L}$ from aqueous solutions, using $\mathrm{R}^{\mathrm{F}}$ paper as a porous membrane that is permeable to air (Fig. S4, ESI $\dagger$ and Movie S4 $\uparrow$ ).

\section{Functional microfluidic devices in paper: serial diluters and 90 droplet generators}

One of the useful characteristics of open-channel PDMS microfluidics is the ability to precisely generate monodisperse microdroplets of immiscible fluids, or well-defined gradients of solutes in miscible liquids. ${ }^{53-55}$ The precise control over volumes 95 and concentration conferred by droplet-based (or digital) microfluidics has led to new avenues of development in chemical and biochemical screening, protein crystallization, enzymatic kinetics, and bioassays. ${ }^{56}$

As proof of principle, we applied our paper engraving 100 technique to fabricate inexpensive devices for performing serial dilutions, and for generating droplets in an immiscible phase. Fig. $6 \mathrm{~A}-\mathrm{B}$ shows the serial dilution of an aqueous solution of $0.05 \%$ Methylene Blue with a solution of $0.05 \%$ Congo Red. Both solutions were provided to their respective inlets at a flow rate of ${ }_{105} 10 \mu \mathrm{L} / \mathrm{min}$ (using a syringe pump). The serial dilution can be visualized as a change in the color of liquids inside the channels and at the outlets, from red and blue to shades of purple.

Fig. 6C-F shows the formation of droplets in a T-junction in a paper microfluidic device with engraved channels $(\sim 300 \mu \mathrm{m} x$ $110200 \mu \mathrm{m}$ cross-sectional dimensions). Hexadecane served as the "carrier" fluid, or continuous phase, and an aqueous solution of dye $(0.05 \%$ Congo Red) served as the dispersed phase. We established the flow of the aqueous solution and the oil with syringe pumps. These two phases met at the junction, where 115 droplets "pinched-off" at regular intervals. 
The size of droplets generated at microfluidic T-junctions can be controlled by adjusting the relative flow rates of the continuous and dispersed phases. ${ }^{57}$ We thus formed droplets of different sizes by varying the flow rate of the aqueous solution 5 while keeping the flow rate of the continuous phase constant (See Movies S2†, S3†, S4†). Fig. 6D-F shows several representative micrographs of the system at different flow rates of the continuous and dispersed phases. The droplets generated in the paper microfluidic device were monodisperse; the coefficient of 10 variation $(\mathrm{CV})$ of the length of the droplets (the distance between the furthest downstream and upstream points along the interface of a fully detached plug) generated at a frequency of $1.25 \mathrm{~Hz}$ was $1.1 \%$ (see discussion in ESI $\dagger$ ). By varying the flow rates of the dispersed and continuous phases, water-in-oil droplets can be 15 generated in engraved channels at frequencies between 0.5 and $10 \mathrm{~Hz}$, similar to typical frequencies obtained in PDMS microfluidic devices. ${ }^{56}$ Frequencies above $10 \mathrm{~Hz}$ are not presently feasible because of limitations on the operating pressures that the devices can withstand $(30 \mathrm{kPa})$.

20 Paper-based droplet microfluidic systems offer several potential advantages over systems fabricated in PDMS. Many of the potential uses of droplet-based microfluidic devices are driven by a need to compartmentalize solutions of particles, cells or molecules into microreactors for further manipulation by the 25 addition (or removal) of other reagents. The high gas permeability of paper facilitates the transfer of volatile reagents from neighboring channels, or from the environment, and the release of gaseous by-products. Other active means to manipulate droplets involve forces produced by electric fields to create, 30 recombine, split and sort charged droplets. ${ }^{58}$ The integration of embedded circuits requires materials with high dielectric strength, able to sustain the high voltages required to charge the oil-water interface. The dielectric strength (breakdown voltage) of the $\mathrm{R}^{\mathrm{F}}$ papers that we tested varied between $8.3 \pm 0.3 \mathrm{MV} / \mathrm{m}$ (cardstock ${ }_{35}$ paper) and $71.4 \pm 8.0 \mathrm{MV} / \mathrm{m}$ (Canson tracing paper-a high density, cellulose paper). For comparison, the dielectric strength of PDMS is $20 \mathrm{MV} / \mathrm{m}^{59}$ Thus, this fabrication technique, when combined with an appropriate choice of paper, surface chemistry, and channel design, might lead to the development of more 40 complex droplet-based microfluidic technologies.

\section{Conclusions}

The combination of cardstock paper silanized with a fluoroalkyl ${ }_{45}$ silane and a craft cutter provides the basis for a simple new bench-top technique for the fabrication of open-channel microfluidic devices. Like the familiar PDMS-based microfluidic systems, these devices have open, microscale $\left(45 \times 100 \mu \mathrm{m}^{2}\right.$ cross section) channels, exhibit pressure-driven, low-Reynolds-number

50 flows and are compatible with multiphase flows involving droplets of water in oil. Like other paper-based microfluidic devices, the devices are inexpensive, mechanically flexible, lightweight, disposable by burning, and highly permeable to gases. These features provide new capabilities that would be ${ }_{55}$ difficult to achieve using other types of microfluidic devices: i) fast exchange of volatile compounds between adjacent, fluidically-independent channels, as well as access to reagents in the vapor phase outside the channel; ii) regulation of flow rate by adjusting the folding angle at a "fold" valve (Fig. 3), or by using ${ }_{60}$ differences in pressure to bridge unconnected channels (Fig. 4); iii) reduced probability of adsorption or exchange of hydrophobic compounds between channel walls in omniphobic paper compared to PDMS, ${ }^{60}$ due to the oleophobicity of the surface; iv) easy reconfiguration of the design of pre-existing microfluidic ${ }_{65}$ devices, as it benefits specific applications, by engraving additional channels or folding to create valves at desired positions; v) compatibility with some liquids that swell or destroy PDMS, including aromatic hydrocarbons (xylenes, toluene, benzene) and concentrated sulfuric acid. ${ }^{60}$

70 These pressure-driven open-channel microfluidic devices have three limitations: i) The omniphobic paper generated by silanization with a fluoroalkane $\left(\mathrm{C}_{10}{ }^{\mathrm{F}}\right)$ is not suitable for manipulating fluids with surface tension below $27 \mathrm{mN} / \mathrm{m}$ (e.g. pentane, methanol) since these fluids will penetrate the pores of 75 the silanized paper (many liquids with low surface tensions are also incompatible with PDMS-based microfluidic systems, as they swell or dissolve the PDMS). ${ }^{60}$ ii) The size of the channels is defined by the thickness of the blade, and it is thus difficult, with a simple cutter with a single blade, to produce continuous ${ }_{80}$ channels with variable widths. iii) The gas permeability of paper can lead to partial evaporation of the liquid, causing changes in the concentration, medium osmolality, or even complete drying of the device. This problem can be addressed using a humiditycontrolled atmosphere, by incorporating hydration channel 85 networks, or by packaging the device.

Pressure-driven paper microfluidic devices fabricated using this technique, with further development, have the potential to provide new functional options in biomedical fluid handling, clinical diagnostics, environmental monitoring, and microfluidic 90 and electronic systems.

\section{Acknowledgements}

We are thankful for the financial support provided by the Bill and Melinda Gates Foundation under award 51308. R.V.M. acknowledges funding by the FP7 People program under the ${ }_{95}$ project Marie Curie IOF-275148. E.J.M. thanks the Natural Sciences and Engineering Research Council of Canada for individual support (NSERC Postdoctoral Fellowship). We thank Dr. Christoph Keplinger Dr. Claudiu Stan and Dr. Barbara Smith for helpful discussions.

100

\section{References}

1. A. W. Martinez, S. T. Phillips, B. J. Wiley, M. Gupta and G. M. Whitesides, Lab Chip, 2008, 8, 2146-2150.

2. E. Carrilho, A. W. Martinez and G. M. Whitesides, Anal. Chem., 105 2009, 81, 7091-7095.

3. L. Y. Yeo, H. C. Chang, P. P. Chan and J. R. Friend, Small, 2011, 7, $12-48$.

4. W. Dungchai, O. Chailapakul and C. S. Henry, Anal. Chem., 2009, 81, 5821-5826.

110 5. X. Li, J. F. Tian, G. Garnier and W. Shen, Colloids and Surfaces BBiointerfaces, 2010, 76, 564-570.

6. K. Abe, K. Suzuki and D. Citterio, Anal Chem, 2008, 80, 6928-6934.

7. X. Li, D. R. Ballerini and W. Shen, Biomicrofluidics, 2012, 6.

8. G. M. Whitesides, Nature, 2006, 442, 368-373.

115 9. A. W. Martinez, S. T. Phillips, G. M. Whitesides and E. Carrilho, Anal. Chem., 2010, 82, 3-10. 
10. S. J. Vella, P. Beattie, R. Cademartiri, A. Laromaine, A. W. Martinez, S. T. Phillips, K. A. Mirica and G. M. Whitesides, Anal. Chem., 2012, 84, 2883-2891.

11. H. Sharma, N. Diep, A. Chen, V. Lew and M. Khine, Ann. Biomed. Eng., 2011, 39, 1313-1327.

12. J. Mairhofer, K. Roppert and P. Ertl, Sensors, 2009, 9, 4804-4823.

13. L. Ge, S. M. Wang, X. R. Song, S. G. Ge and J. H. Yu, Lab Chip, 2012, 12, 3150-3158

14. X. X. Yang, O. Forouzan, T. P. Brown and S. S. Shevkoplyas, Lab Chip, 2012, 12, 274-280.

15. M. S. Khan, G. Thouas, W. Shen, G. Whyte and G. Garnier, Anal. Chem., 2010, 82, 4158-4164.

16. H. Gardeniers and A. Van den Berg, Int. J. Environ. Anal. Chem., 2004, 84, 809-819.

15 17. L. Marle and G. M. Greenway, TrAC-Trends in Analytical Chemistry, 2005, 24, 795-802.

18. A. J. Hopwood, C. Hurth, J. N. Yang, Z. Cai, N. Moran, J. G. LeeEdghill, A. Nordquist, R. Lenigk, M. D. Estes, J. P. Haley, C. R. McAlister, X. Chen, C. Brooks, S. Smith, K. Elliott, P. Koumi, F.

20 Zenhausern and G. Tully, Anal. Chem., 2010, 82, 6991-6999.

19. P. Liu, X. Li, S. A. Greenspoon, J. R. Scherer and R. A. Mathies, Lab Chip, 2011, 11, 1041-1048.

20. G. Lin and A. P. Lee, in Foods for Health in the 21st Century: A Road Map for the Future, eds. M. E. Gershwin and M. R. C. Greenwood, 2010, vol. 1190, pp. 186-192.

21. A. C. Glavan, R. V. Martinez, A. B. Subramaniam, H. J. Yoon, R. M. D. Nunes, H. Lange, M. M. Thuo and G. M. Whitesides., Adv. Funct. Mater., 2013 (submitted).

22. A. L. Liu, F. Y. He, Y. L. Hu and X. H. Xia, Talanta, 2006, 13031308.

23. B. Weigl, R. Bardell, T. Schulte, F. Battrell and J. Hayenga, Biomedical Microdevices, 2001, 3, 267-274.

24. L. W. Luo, C. Y. Teo, W. L. Ong, K. C. Tang, L. F. Cheow and L. Yobas, Journal of Micromechanics and Microengineering, 2007, 17, N107-N111.

25. D. A. Bartholomeusz, R. W. Boutte and J. D. Andrade, Journal of Microelectromechanical Systems, 2005, 14, 1364-1374.

26. P. K. Yuen and V. N. Goral, Lab Chip, 2010, 384-387.

27. D. Zahner, J. Abagat, F. Svec, J. M. Frechet and P. A. Levkin, Adv. Mater., 2011, 23, 3030-3034.

28. H. Becker and U. Heim, Sensors and Actuators A-Physical, 2000, 83, 130-135.

29. C. D. Chin, Y. K. Cheung, T. Laksanasopin, M. M. Modena, S. Y. Chin, A. A. Sridhara, D. Steinmiller, V. Linder, J. Mushingantahe, G.

45 Umviligihozo, E. Karita, L. Mwambarangwe, S. L. Braunstein, J. van de Wijgert, R. Sahabo, J. E. Justman, W. El-Sadr and S. K. Sia, Clin. Chem., 2013.

30. C. D. Chin, T. Laksanasopin, Y. K. Cheung, D. Steinmiller, V. Linder, H. Parsa, J. Wang, H. Moore, R. Rouse, G. Umviligihozo, E. Karita, L. Mwambarangwe, S. L. Braunstein, J. van de Wijgert, R. Sahabo, J. E. Justman, W. El-Sadr and S. K. Sia, Nat Med, 2011, 17, 1015-1019.

31. C. D. Chin, V. Linder and S. K. Sia, Lab Chip, 2012, 12, 2118-2134.

32. A. W. Martinez, S. T. Phillips, M. J. Butte and G. M. Whitesides, Angew. Chem., 2007, 46, 1318-1320.

33. A. W. Martinez, S. T. Phillips, E. Carrilho, S. W. Thomas, H. Sindi and G. M. Whitesides, Anal. Chem., 2008, 80, 3699-3707.

34. A. W. Martinez, S. T. Phillips and G. M. Whitesides, Proc. Natl. Acad. Sci. U. S. A., 2008, 105, 19606-19611.

60 35. E. Carrilho, S. T. Phillips, S. J. Vella, A. W. Martinez and G. M. Whitesides, Anal. Chem., 2009, 81, 5990-5998.

36. D. A. Bruzewicz, M. Reches and G. M. Whitesides, Anal. Chem., 2008, 80, 3387-3392.

37. X. Li, J. Tian, T. Nguyen and W. Shen, Anal. Chem., 2008, 80, 91319134.

38. G. Chitnis, Z. Ding, C. L. Chang, C. A. Savran and B. Ziaie, Lab Chip, 2011, 11, 1161-1165.

39. E. M. Fenton, M. R. Mascarenas, G. P. Lopez and S. S. Sibbett, ACS Appl. Mater. Inter., 2009, 1, 124-129.
70 40. S. Mendez, E. M. Fenton, G. R. Gallegos, D. N. Petsev, S. S. Sibbett, H. A. Stone, Y. Zhang and G. P. Lopez, Langmuir, 2010, 26, 13801385.

41. W. Wang, W. Y. Wu and J. J. Zhu, J. Chromatogr. A, 2010, 1217, 3896-3899.

75 42. P. Kauffman, E. Fu, B. Lutz and P. Yager, Lab Chip, 2010, 10, 2614 2617.

43. B. R. Lutz, P. Trinh, C. Ball, E. Fu and P. Yager, Lab Chip, 2011, 11, 4274-4278.

44. L. Ge, J. Yan, X. Song, M. Yan, S. Ge and J. Yu, Biomaterials, 2012, 33, $1024-1031$

45. G. J. Worsley, S. L. Attree, J. E. Noble and A. M. Horgan, Biosens. Bioelectron., 2012, 34, 215-220.

46. Y. K. Oh, H. A. Joung, S. Kim and M. G. Kim, Lab Chip, 2013, 13, 768-772.

85 47. R. F. Ismagilov, A. D. Stroock, P. J. A. Kenis, G. Whitesides and H. A. Stone, Appl. Phys. Lett., 2000, 76, 2376-2378.

48. L. Capretto, W. Cheng, M. Hill and X. L. Zhang, Microfluidics: Technologies and Applications, 2011, 304, 27-68.

49. D. B. Weibel, M. Kruithof, S. Potenta, S. K. Sia, A. Lee and G. M. Whitesides, Anal. Chem., 2005, 77, 4726-4733.

50. T. Thorsen, S. J. Maerkl and S. R. Quake, Science, 2002, 298, 580584 .

51. M. A. Unger, H. P. Chou, T. Thorsen, A. Scherer and S. R. Quake, Science, 2000, 288, 113-116.

95 52. L. Pal, M. K. Joyce and P. D. Fleming, TAPPI J., 2006, 5, 10-16.

53. H. Song, D. L. Chen and R. F. Ismagilov, Angew. Chem., 2006, 45, 7336-7356.

54. P. Garstecki, M. J. Fuerstman, H. A. Stone and G. M. Whitesides, Lab Chip, 2006, 6, 437-446.

100 55. A. S. Utada, E. Lorenceau, D. R. Link, P. D. Kaplan, H. A. Stone and D. A. Weitz, Science, 2005, 308, 537-541.

56. S. Y. Teh, R. Lin, L. H. Hung and A. P. Lee, Lab Chip, 2008, 8, 198 220.

57. S. K. Y. Tang and G. M. Whitesides, in Optofluidics: Fundamentals, Devices, and Applications, eds. F. Yeshaiahu, P. Demetri and Y. Changhuei, McGraw Hill Professional, Access Engineering, 2010, ch. 2: Basic Microfluidic and Soft Lithographic Techniques.

58. G. F. B. Christopher, Manipulation of Droplets in Microfluidic Channels Using T-junction Geometry, Carnegie Mellon University, 2008.

59. J. C. McDonald and G. M. Whitesides, Acc. Chem. Res., 2002, 35, 491-499.

60. J. N. Lee, C. Park and G. M. Whitesides, Anal. Chem., 2003, 75, 6544-6554.

115 61. L. L. Spiller, Anal. Lett., 1989, 22, 2561-2573.

62. B. Z. Shakhashiri, Chemical Demonstrations, Volume 2: A Handbook for Teachers of Chemistry, University of Wisconsin Press, 1985.

63. N. Zamel, X. G. Li and J. Shen, Energy Fuels, 2009, 23, 6070-6078.

64. J. J. Kester and O. Fennema, J. Am. Oil Chem. Soc., 1989, 66, 11391146.

65. W. L. Robb, Ann. N. Y. Acad. Sci., 1968, 146, 119-137. 